\title{
Study on the Porosity of Saturated Fragmentized Coals during Creep Process and Constitutive Relation
}

\author{
Peng Gong $\mathbb{D},{ }^{1,2}$ Zhanguo Ma $\mathbb{i}$, ${ }^{1,2}$ Yongheng Chen $\mathbb{D}^{2},{ }^{2}$ Shixing Cheng $\mathbb{D},{ }^{1}$ and Kelong Li $\mathbb{i D}^{2}$ \\ ${ }^{1}$ State Key Laboratory for Geomechanics and Deep Underground Engineering, China University of Mining and Technology, \\ Xuzhou, Jiangsu 221116, China \\ ${ }^{2}$ School of Mechanics and Civil Engineering, China University of Mining and Technology, Xuzhou, Jiangsu 221116, China
}

Correspondence should be addressed to Zhanguo Ma; 1044@cumt.edu.cn

Received 23 June 2020; Revised 7 September 2020; Accepted 25 September 2020; Published 19 October 2020

Academic Editor: Hailing Kong

Copyright (C) 2020 Peng Gong et al. This is an open access article distributed under the Creative Commons Attribution License, which permits unrestricted use, distribution, and reproduction in any medium, provided the original work is properly cited.

\begin{abstract}
Pore abundance and deformation characteristics of saturated fragmentized coals during creep process are of significant meaning to the study on ground sediment in the mined-out area. The law of porosity variation of saturated fragmentized coals during creep process and its creep constitutive model were studied by using the self-developed multiphase coupling creep test device. And, results have indicated that the porosity logarithm of fragmentized coal during creep process shows a linear negative correlation with the time $\ln (n-a)=-c t+\ln b$, and the porosity decrease is evidently divided into three phases. In addition, when the stress level is relatively low, the porosity decreases slowly; when the stress level rises up, the porosity decreases quickly; when the stress level remains stable finally, the porosity is smaller. Under the equal stress, as the grain size of fragmentized coals decreases, the porosity tends to decrease, and as the grain size of fragmentized coal tends to be stable, the porosity tends to increase; the creep constitutive equation of fragmentized coals with different grain sizes was established by using the Kelvin-Voigt model, and the correlation analysis shows that the Kelvin-Voigt creep model of fragmentized coals is reasonable.
\end{abstract}

\section{Introduction}

Coal is an inhomogeneous and porous media [1], and thus compaction and bearing problems of fragmentized coals are quite common during coal mining. With the impact of external forces, coals are constantly crushed, densified, and compacted; meanwhile, some relevant physical and mechanical properties of fragmentized coals are changed, which can cause some engineering problems such as overburden movement, ground sediment [2-5], and side slope instability in the mined-out area. The study on the law of porosity variation of fragmentized coals during creep process [6-11] and its creep constitutive equation lays a theoretical foundation for resolving engineering problems such as the evaluation of deformation of ground sediment in the minedout area. But, among current analyses of fragmentized rocks [12-14], few can systematically analyze the porosity variation law of fragmentized rocks during creep in terms of different stress and different grain sizes; in the description of creep deformation law, regression analysis means, such as logarithm fitting and fit exponential decay, are usually adopted, but its constitutive relation is not analyzed, and its creep deformation mechanism is explained from the perspective of the material prosperity of fragmentized rocks.

\section{Test Equipment and Test Methods}

2.1. Coal Sample. In order to study the law of porosity variation of fragmentized coals with different grain sizes under different loads during compaction and creep process, fragmentized coals with five grain sizes are selected, whose uniaxial compressive strength is $15 \mathrm{MPa}$. Their grades and sizes are shown in Table 1, and fragmentized coals and preparation instruments are shown in Figure 1(a).

2.2. Test Equipment. It is easy for loose and fragmentized coals without confining pressure to flow transversely under axial load; thus, they cannot bear heavy loads, but fragmentized coals in special sites such as the roadway of the coal 
Table 1: Grades and sizes of fragmentized coals.

\begin{tabular}{lcc}
\hline Grades & Grade symbol & Size $(\mathrm{mm})$ \\
\hline 1 & $\mathrm{~m} 1$ & $20.0 \sim 25.0$ \\
2 & $\mathrm{~m} 2$ & $15.0 \sim 20.0$ \\
3 & $\mathrm{~m} 3$ & $10.0 \sim 15.0$ \\
4 & $\mathrm{~m} 4$ & $5.0 \sim 10.0$ \\
5 & $\mathrm{~m} 5$ & $2.5 \sim 5.0$ \\
\hline
\end{tabular}

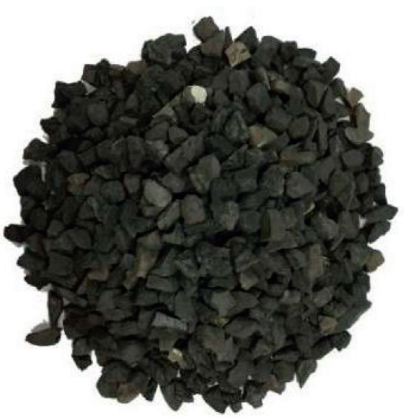

(a)

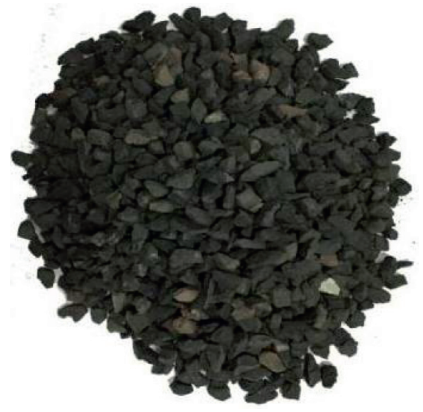

(b)

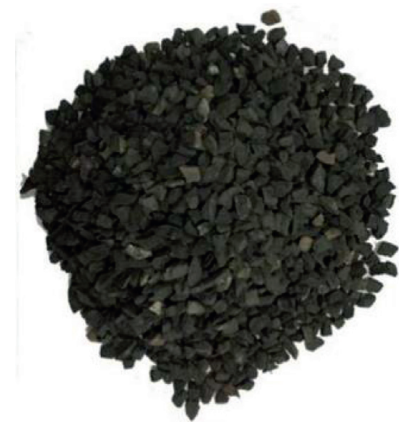

(c)

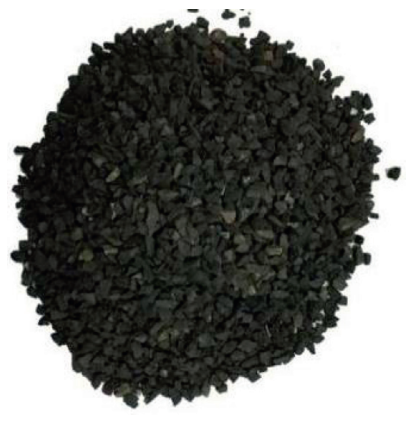

(d)

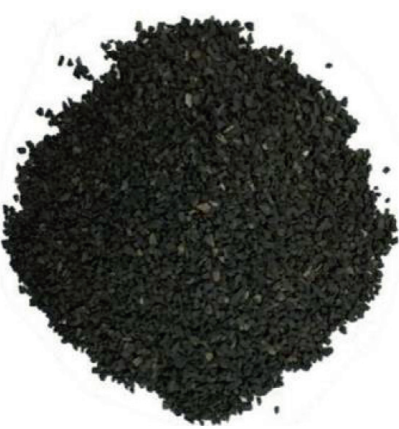

(e)

FIgURE 1: Classification of particle size: (a) $20.0 \sim 25.0 \mathrm{~mm}$. (b) $15.0 \sim 20.0 \mathrm{~mm}$. (c) $10.0 \sim 15.0 \mathrm{~mm}$. (d) $5.0 \sim 10.0 \mathrm{~mm}$. (e) $2.5 \sim 5.0 \mathrm{~mm}$.

mining face have strong confining pressure. In consideration of this, the self-developed multiphase coupling creep test device of fragmentized coals is designed which applies the leadscrew system to provide loads, as shown in Figure 1(b). When it reaches certain pressure, the power supply can be cut off, and it relies on leadscrew self-locking to provide pressure to avoid too much energy consumption during the long-time test. The deformation test adopts an FT81 displacement sensor and an LVDV-3 digital indicator. In addition, one spherical hinge needs to be added above the pressure-bearing deformation instrument to avoid unbalance loading due to pedestal or spring imbalance.

The assembly height of the compaction apparatus of fragmentized coals is $300 \mathrm{~mm}$ ( $140 \mathrm{~mm}$ high piston and $170 \mathrm{~mm}$ long cylinder barrel). The inner diameter of the cylinder barrel is $100 \mathrm{~mm}$, and the wall thickness is $15 \mathrm{~mm}$; during processing, ordinary No. 45 steel is fully quenched to enhance hardness and prevent fragmentized coal edges from scratching the inner wall and increasing the resistance; cylinder barrel bottom and pedestal are linked by bolts. The maximum axial loading pressure designed for the deformation instrument is $180 \mathrm{MPa}$.
2.3. Test Methods. Firstly, the deformation instrument is assembled and filled with the sample of statured fragmentized coals to be tested. In consideration of the displacement instrument stroke and the estimated maximum deformation, those uncompressed fragmentized rocks are controlled to be $128 \mathrm{~mm}$ high and are slightly shaken to be dense for each time; finally, they are divided into two groups for test under the load of 8 and $12 \mathrm{Mpa}$, respectively.

Test procedures are as follows: the sample of saturated fragmentized coals is loaded to the working load, and the loading process is recorded; the saturated drip is opened, and water is fed to the compaction apparatus at the appropriate time under the symphonic effect to maintain the coal sample always under the saturation state; each group of deformation instruments provides two sets of displacement testing systems, and the displacement reading is the average value; the load is observed daily in a fixed time interval, the load is compensated when necessary, and the displacement data and time are recorded. Each type of test is divided into 3 5 groups, and the average value of test data is the final result. For test schemes with results of significant dispersion, test groups are added until three groups draw the similar conclusion. 


\section{The Law of Porosity Variation with Time}

Porosity refers to the ratio of pore volume between particles to total volume of fragmentized media, namely,

$$
n=\frac{V_{s}-V_{z}}{V_{s z}}
$$

where $n$ denotes the porosity of fragmentized coals, $V_{s z}$ denotes the total volume of fragmentized coals before test, $V_{s}$ denotes the total volume of fragmentized coals during test, and $V_{z}$ denotes the volume of solid particles in fragmentized coals.

\subsection{Influence of Stress Level on the Porosity of Fragmentized} Coals. Stress level produces a significant impact on the porosity of fragmentized coals, and the relation curve between the porosity of fragmentized coals and the time under different stress levels is obtained from two groups of tests under different loads, as shown in Figures 2 3.

It can be seen from Figure 2 that under the stress of $8 \mathrm{Mpa}$, the porosity variation of coal samples with different grain sizes is periodic. When Grain Size 1 plummets at $69.12 \times 10^{4}(\mathrm{~s})$, the porosity decreases to 0.15466 ; when Grain Size 2 plummets at $60.48 \times 10^{4}(\mathrm{~s})$, the porosity decreases to 0.18585 , and when it plummets at $86.4 \times 10^{4}(\mathrm{~s})$ for the second time, the porosity decreases to 0.18562 ; when Grain Size 3 plummets at $51.84 \times 10^{4}(\mathrm{~s})$, the porosity decreases to 0.16238 , and when it plummets at $69.12 \times 10^{4}(\mathrm{~s})$ for the second time, the porosity decreases to 0.16227 ; when Grain Size 4 plummets at $51.84 \times 10^{4}(\mathrm{~s})$, the porosity decreases to 0.170 80; when Grain Size 5 plummets at $51.84 \times 10^{4}(\mathrm{~s})$, the porosity decreases to 0.21209 . The above phenomena are difficult to observe in the short-time creep test.

It can be seen from Figure 3 that under the stress of $12 \mathrm{Mpa}$, the porosity variation of coal samples with different grain sizes is periodic. When Grain Size 1 plummets at $34.56 \times 10^{4}(\mathrm{~s})$, the porosity decreases to 0.11220 , when it plummets at $51.84 \times 10^{4}(\mathrm{~s})$ for the second time, the porosity decreases to 0.11206 , and when it plummets at $103.68 \times 10^{4}$ (s) for the third time, the porosity decreases to 0.11177 ; when Grain Size 2 plummets at $34.56 \times 10^{4}$ (s), the porosity decreases to 0.17402 , and when it plummets at $51.84 \times 10^{4}$ (s) for the second time, the porosity decreases to 0.17368 ; when Grain Size 3 plummets at $17.28 \times 10^{4}(\mathrm{~s})$, the porosity decreases to 0.12284 , and when it plummets at $69.12 \times 10^{4}$ (s) for the second time, the porosity decreases to 0.12225 ; when Grain Size 4 plummets at $34.56 \times 10^{4}(\mathrm{~s})$, the porosity decreases to 0.140 31; when Grain Size 5 plummets at $43.2 \times 10^{4}(\mathrm{~s})$, the porosity decreases to 0.17252 , and when it plummets at $60.48 \times 10^{4}(\mathrm{~s})$ for the second time, the porosity decreases to 0.17225 .

During the creep of fragmentized coals, the decrease in the porosity of fragmentized coals with five grain sizes is basically divided into three phases. Phase I: the porosity varies quickly and tends to decrease; in this phase, slippage, malposition, and deformation occur between particles of fragmentized coals. Due to the large void between coal particles, the apparent volume decreases quickly, and then the porosity reduces quickly.

Phase II: the porosity changes very quickly and tends to slow down; in this phase, slippage and malposition seldom happen to fragmentized coal particles, and the fragmentized coal particles form a self-supporting structure; as the stress continues to be loaded, under the squeezing impact, (1) particle surface edges are fragmentized, and internal gap diffuses and forms fractures which expand and cause deformation. (2) Some particles slide slightly and cause plastic flow.

Phase III: the porosity changes more slowly than the first two phases, and gradually tends to be stable; due to the attrition crushing in Phase II, a larger gap exists between coal particles and is filled with fine coal particles after fragmentized. As the stress continues to be loaded, the gap between particles becomes smaller and is difficult to be filled; thus, the entire state of coal particles tends to be a critical stable skeleton structure, and deformation tends to be stable.

As shown by the curve, the porosity of fragmentized coals varies with time under two different stresses; when the stress is $8 \mathrm{MPa}$, the porosity decrease corresponding to Grain Sizes $1-5$ in the entire creep process is $5.44 \%, 10.19 \%, 6.45 \%$, $6.64 \%$, and $2.34 \%$, respectively; when the stress is $12 \mathrm{MPa}$, the porosity decrease corresponding to Grain Sizes $1-5$ in the entire creep process is $4.41 \%, 7.89 \%, 8.11 \%, 1.94 \%$, and $4.00 \%$, respectively. When the stress increases from $8 \mathrm{MPa}$ to $12 \mathrm{MPa}$, the porosity decrease corresponding to Grain Sizes $1 \sim 5$ is $27.73 \%, 6.43 \%, 24.71 \%, 17.85 \%$, and $18.78 \%$, respectively. In case of lower load level, slippage and malposition seldom happen to fragmentized coal particles, and fragmentized coal particles can form a self-supporting structure earlier because there are few particle surface edges fracture, internal fracture expansion, and particles plastic flow, and slower porosity decreases due to weak squeezing effect between fragmentized coal particles; as the stress level rises up, more slippage and malposition happen to fragmentized coal particles, and fragmentized coal particles form a self-supporting structure late because there are more particle surface edges fracture, internal fracture expansion, and particles plastic flow due to strong squeezing effect between fragmentized coal particles, so that the porosity decreases quickly, and finally stabilized porosity is smaller.

\subsection{Influence of Grain Size on Porosity of Fragmentized Coals.} Grain size produces a significant impact on the porosity of fragmentized coals, and the relation curve between the porosity of fragmentized coals with different grain sizes and time under different stress levels is obtained from tests, as shown in Figure 4.

Figure 5(a) shows that under the stress of $8 \mathrm{MPa}$, the porosity decreases quickly at early creep, and at $8.64 \times 10^{4}$ (s), the porosity of samples corresponding to Grain Sizes 1 5 is $0.163562,0.206678,0.173566,0.182943$, and 0.217181 , respectively. Afterwards, it goes through three stages of obvious decay. In Phase I until $34.56 \times 10^{4}(\mathrm{~s})$, the porosity of samples corresponding to Grain Sizes 1 5 decreases to 0.155 $049,0.188101,0.163554,0.172351$, and 0.212414 , 


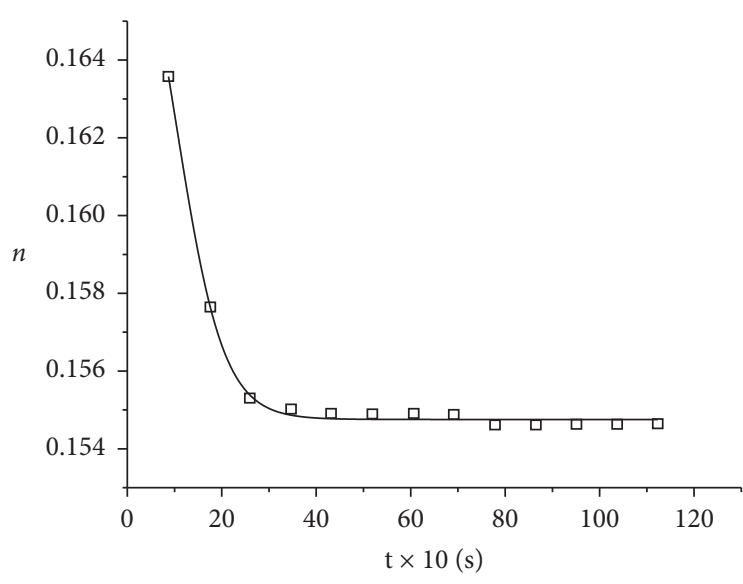

(a)

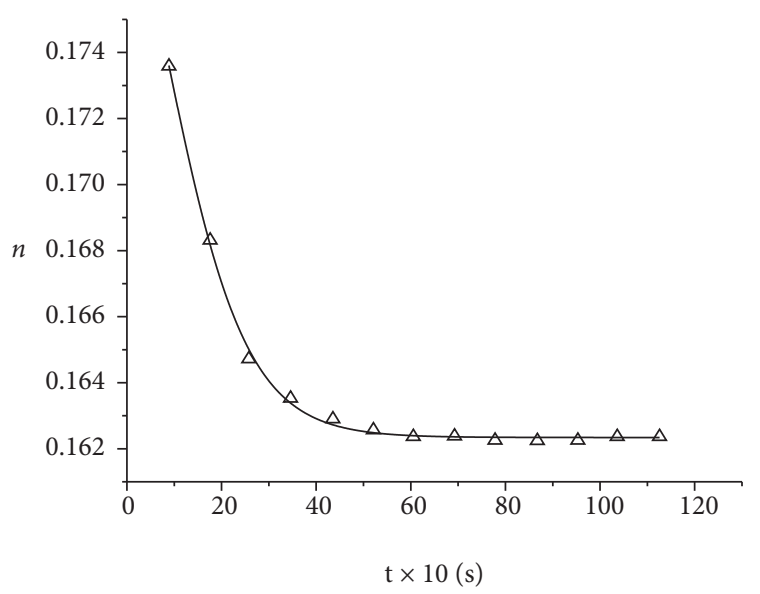

(c)

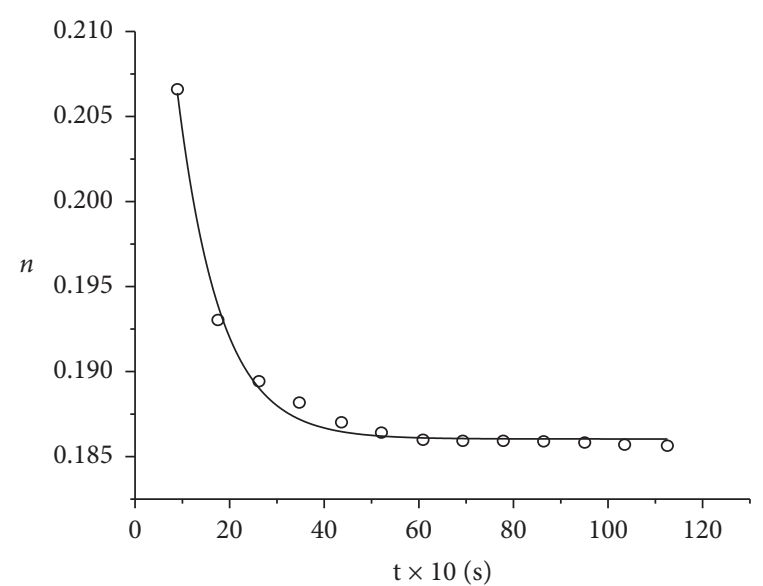

(b)

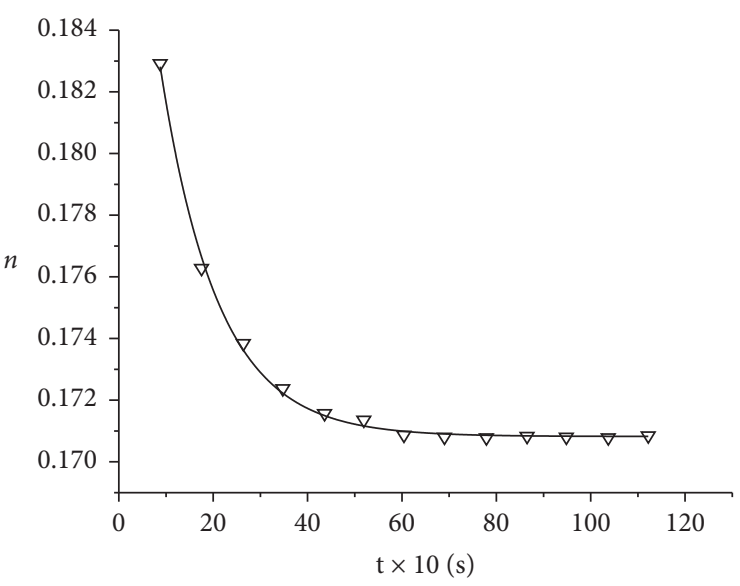

(d)

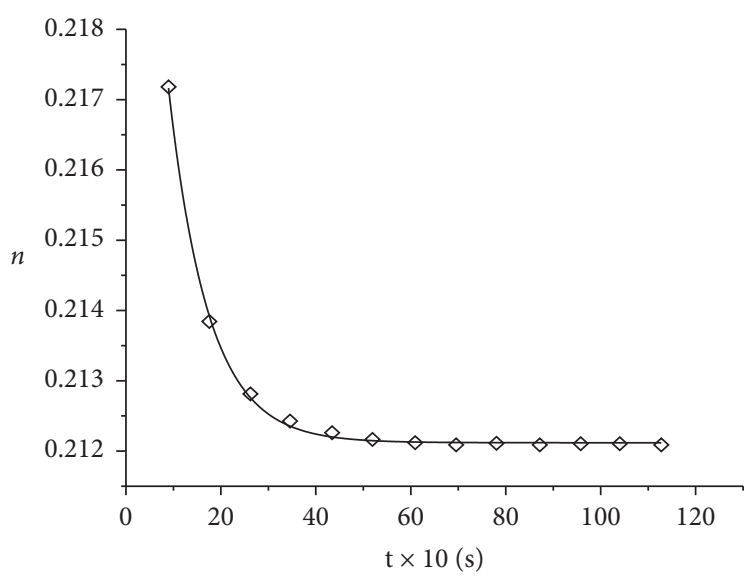

(e)

FiguRE 2: Relation curves between porosity of fragmentized coals and time under the stress of $8 \mathrm{MPa}$. (a) m1. (b) m2. (c) m3. (d) m4. (e) m5.

respectively; in Phase II until $51.84 \times 10^{4}(\mathrm{~s})$, the porosity of samples corresponding to Grain Sizes $1 \sim 5$ decreases to 0.154 919, $0.186297,0.162593,0.171318$, and 0.212174 , respectively; in Phase III until $112.32 \times 10^{4}(\mathrm{~s})$, the porosity of samples corresponding to Grain Sizes 1 5 decreases to 0.154 658, $0.185619,0.162379,0.170800$, and 0.212094 . Targeted at the porosity of the first catastrophe point, the porosity of samples corresponding to Grain Sizes 2 5 increases by $21.32 \%, 5.49 \%, 11.16 \%$, and $37.00 \%$, respectively.

Figure 5(b) shows that under the stress of $12 \mathrm{MPa}$, the porosity decreases quickly at early creep, and at $8.64 \times 10^{4}$ (s), the porosity of samples corresponding to Grain Sizes 1 5 


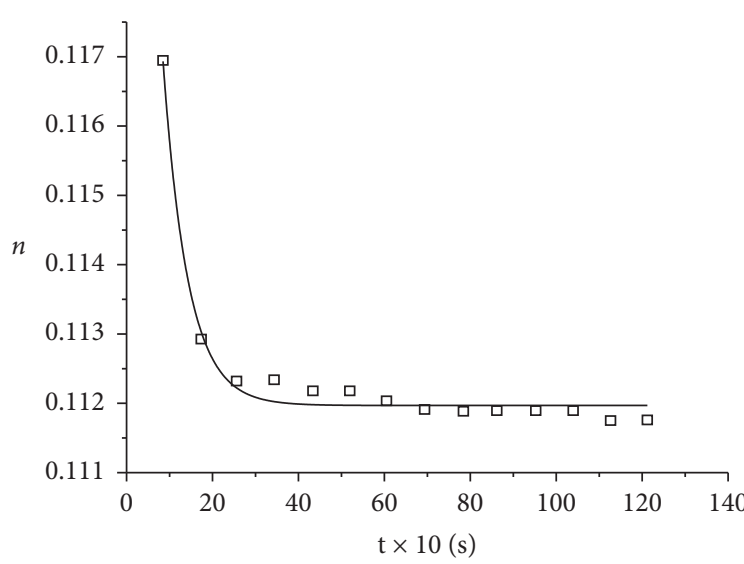

(a)

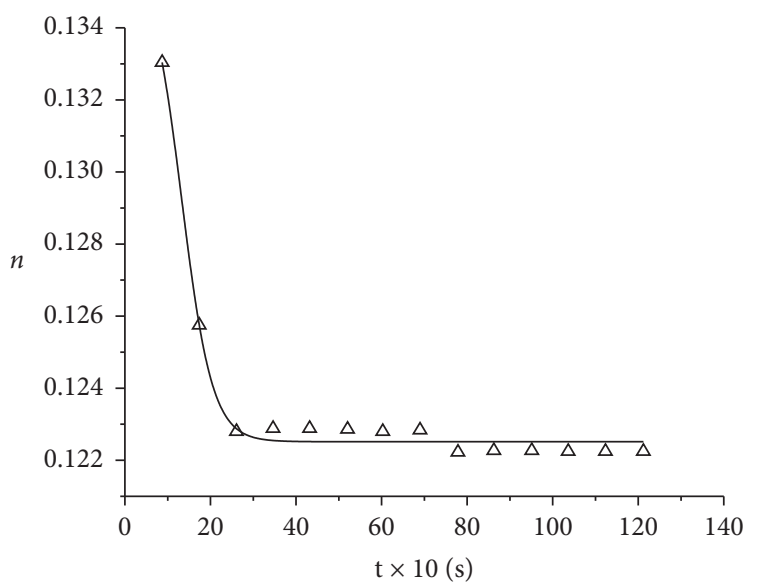

(c)

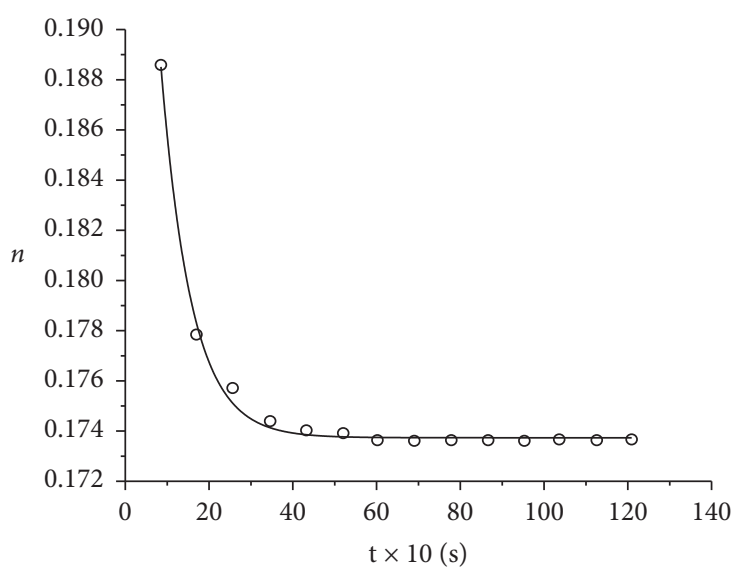

(b)

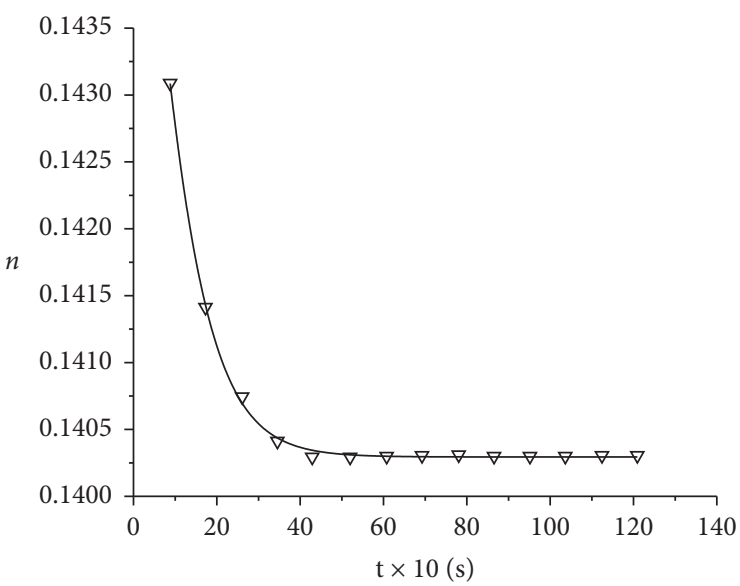

(d)

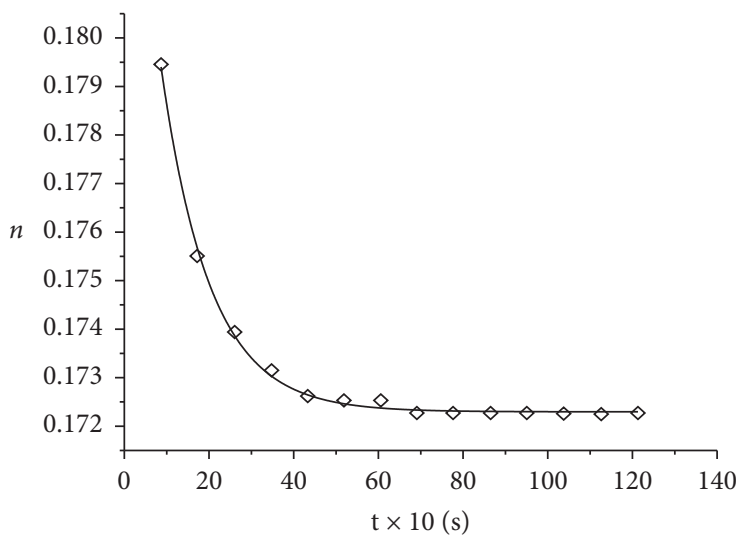

(e)

FIGURE 3: Relation curves between the porosity of fragmentized coals and time under the stress of 12 MPa. (a) m1. (b) m2. (c) m3. (d) m4. (e) m5.

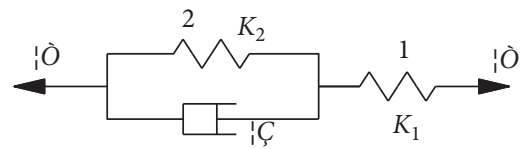

Figure 4: Kelvin-Voigt model. 1, Hook model; 2, Kelvin Model. 


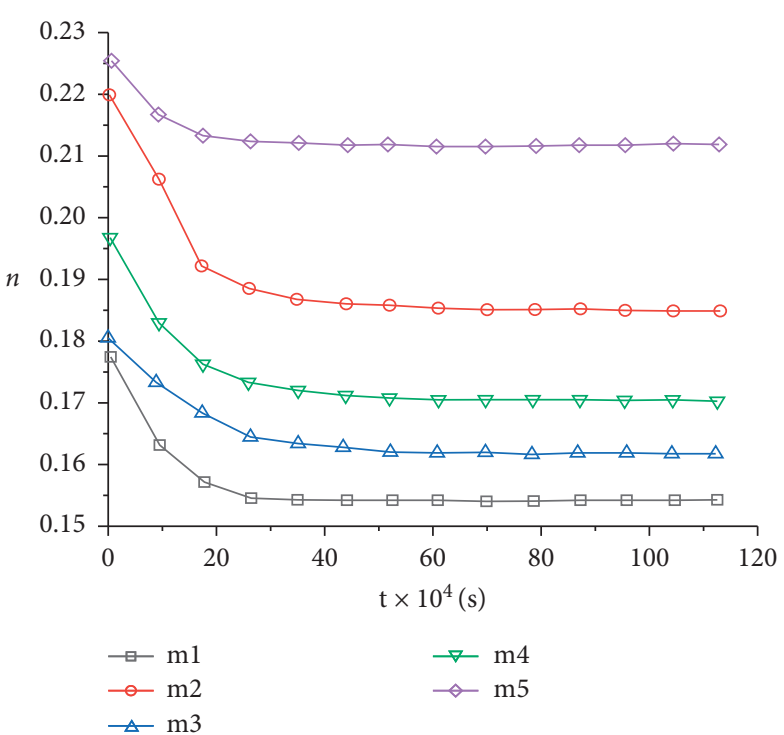

(a)

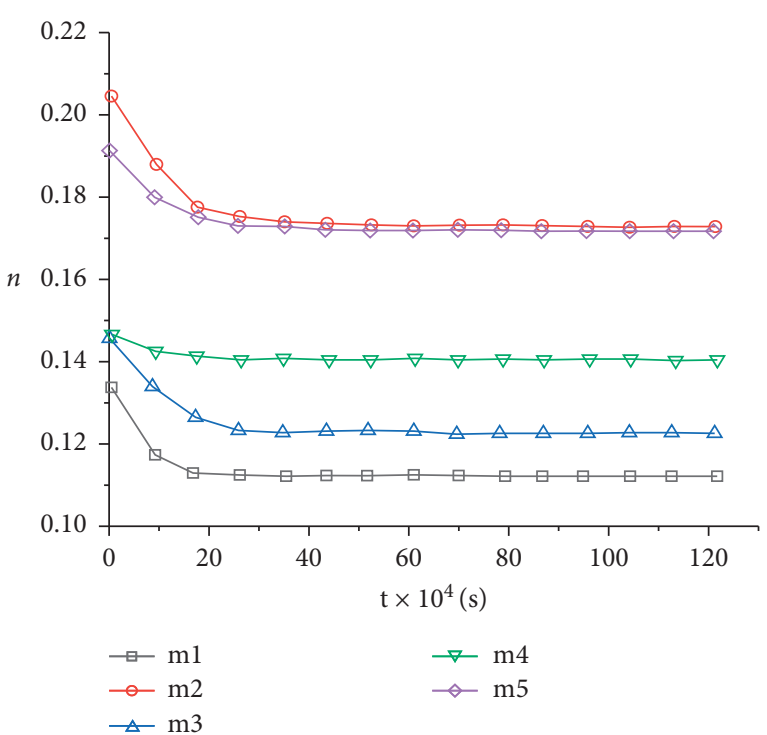

(b)

Figure 5: Influence of grain size on the porosity of fragmentized coals. (a) $8 \mathrm{MPa}$. (b) $12 \mathrm{MPa}$.

is $0.116921,0.188551,0.133041,0.143082$, and 0.179431 , respectively. Afterwards, it goes through three stages of obvious decay. In Phase I until $34.56 \times 10^{4}(\mathrm{~s})$, the porosity of samples corresponding to Grain Sizes 1 5 decreases to 0.112 $343,0.174373,0.122839,0.140419$, and 0.173135 , respectively; in Phase II until $51.84 \times 10^{4}(\mathrm{~s})$, the porosity of samples corresponding to Grain Sizes $1 \sim 5$ decreases to 0.112 199, 0.173 908, $0.122614,0.140308$, and 0.172518 , respectively; in Phase III until $120.91 \times 10^{4}(\mathrm{~s})$, the porosity of samples corresponding to Grain Sizes 1 5 decreases to 0.111 767, $0.173675,0.122$ 252, 0.140308 , and 0.172 253. Targeted at the porosity of the first catastrophe point, the porosity of samples corresponding to Grain Sizes 2 5 increases by $55.22 \%, 9.34 \%, 24.99 \%$, and $54.11 \%$, respectively.

Under the equal stress, as the grain size of fragmentized coal particles decreases, the porosity tends to decrease; as the grain size of fragmentized coal particles tends to be stable, the porosity tends to increase.

3.3. Regression Analysis of the Porosity Variation with Time. According to the regression analysis of the porosity variation of fragmentized coals with five grain sizes and under two stresses with time, the regression equation and relation coefficient are obtained and shown in Table 2.

Under two stresses, the porosity logarithm of fragmentized coals during creep process shows a linear negative correlation with the time, namely,

$$
\ln (n-a)=-c t+\ln b
$$

where $n$ denotes the porosity of fragmentized coals; $t$ denotes the time; $a, b$, and $c$ are the regression coefficients. As the creep time increases, the $a$ value infinitely nears the final porosity after creep. Under the stress of $8 \mathrm{MPa}$, the $a$ value of Grain Sizes 1 5 corresponding to samples is $0.1548,0.1860$, $0.1623,0.1708$, and 0.2121 , respectively, which tends to increase with the decrease in grain size; under the stress of $12 \mathrm{MPa}$, the $a$ value of Grain Sizes $1 \sim 5$ corresponding to samples is $0.1120,0.1738,0.1225,0.1403$, and 0.1723 , respectively, which tends to increase with the decrease in grain size. The $a$ value tends to increase with the decrease in grain size and tends to decrease with the increase in load.

\section{Creep Constitutive Model of Saturated Fragmentized Coals}

The creeping property of fragmentized coals refers to the long-term mechanical effect suffered by fragmentized coals. The creeping property and law of fragmentized coals can be explained by establishing the creep constitutive equation. It can be found from the analysis of test results that early instantaneous strain and deformation limit of long-term creep exist during the entire creep test process of fragmentized coals. Therefore, Kelvin-Voigt is selected to describe the creep law of fragmentized coals and disclose its constitutive relation. A correlation analysis is conducted on the Kelvin-Voigt model, and a comparison is conducted with the previously common fitting curve of the first index decay to verify the reasonability of creep constitutive relation of fragmentized coals described by the Kelvin-Voigt model.

4.1. Model Building of Creep Constitutive Model of Fragmentized Coals. In broad sense, the Kelvin-Voigt model of the creep constitutive model is shown in Figure 4.

Under the condition of applying the constant load $\sigma_{0}=\sigma_{1}=\sigma_{2}$,

$$
\varepsilon_{1}=\varepsilon_{2}=\varepsilon_{3}=\frac{\sigma_{0}}{K_{1}}+\frac{\sigma_{0}}{K_{2}}\left[1-\exp \left(-\frac{K_{2}}{\eta} t\right)\right] \text {. }
$$

When $t=0, \varepsilon_{0}=\sigma_{0} / K_{1}$, where $\varepsilon_{0}$ is the instantaneous deformation, which is independent from time and is realized by the $\mathrm{H}$ element (element 1 ). 
TABLE 2: Fitted equation coefficients of fragmentized coals under different axial stresses.

\begin{tabular}{|c|c|c|c|}
\hline Grain size (in $\mathrm{mm}$ ) & Regression coefficient & Axial stress: $8 \mathrm{MPa}$ & Axial stress: $12 \mathrm{MPa}$ \\
\hline \multirow{3}{*}{ m1 (20-25) } & A & 0.1548 & 0.1120 \\
\hline & $\mathrm{B}$ & 0.2839 & 0.0233 \\
\hline & $\mathrm{C}$ & 0.0676 & 0.1800 \\
\hline \multirow{3}{*}{$\mathrm{m} 2(15-20)$} & $\mathrm{A}$ & 0.1860 & 0.1738 \\
\hline & $\mathrm{B}$ & 0.0538 & 0.0483 \\
\hline & $\mathrm{C}$ & 0.1118 & 0.1375 \\
\hline \multirow{3}{*}{ m3 (10-15) } & $\mathrm{A}$ & 0.1623 & 0.1225 \\
\hline & $\mathrm{B}$ & 0.0231 & 0.0365 \\
\hline & $\mathrm{C}$ & 0.0815 & 0.1430 \\
\hline \multirow{3}{*}{$\mathrm{m} 4(5-10)$} & $\mathrm{A}$ & 0.1708 & 0.1403 \\
\hline & $\mathrm{B}$ & 0.0250 & 0.0071 \\
\hline & $\mathrm{C}$ & 0.0845 & 0.1075 \\
\hline \multirow{3}{*}{ m5 (2.5-5) } & $\mathrm{A}$ & 0.2121 & 0.1723 \\
\hline & $\mathrm{B}$ & 0.0141 & 0.0150 \\
\hline & $\mathrm{C}$ & 0.1187 & 0.0865 \\
\hline
\end{tabular}

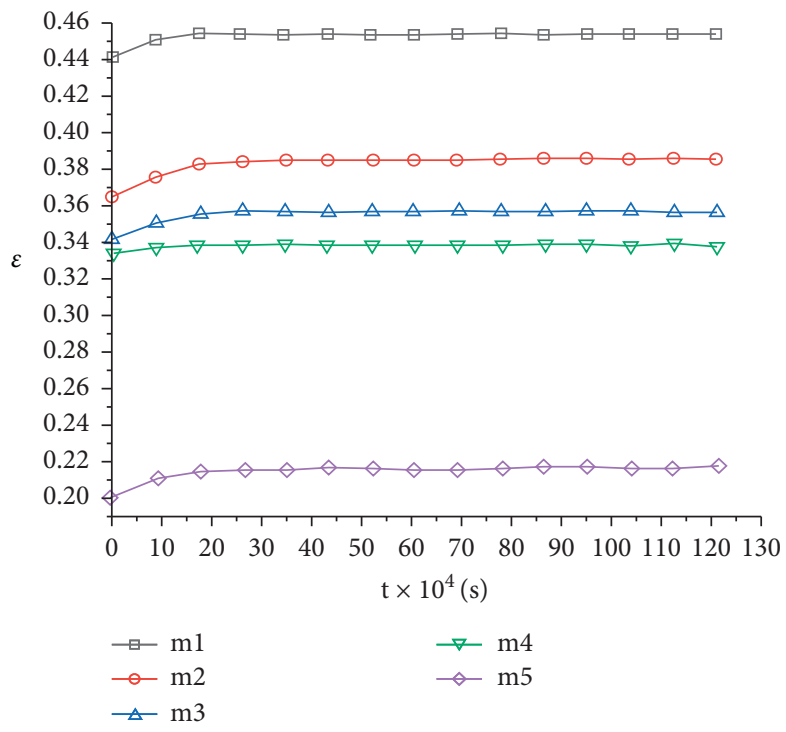

FIGURE 6: The $\varepsilon$-t relation of fragmentized coals with different grain sizes under the stress of $12 \mathrm{MPa}$.

TABLE 3: Kelvin-Voigt model parameter table of fragmentized coals with different grain sizes under the stress of $12 \mathrm{MPa}$.

\begin{tabular}{|c|c|c|c|c|c|}
\hline \multirow{2}{*}{ Parameter } & \multicolumn{5}{|c|}{ Grain size } \\
\hline & $\mathrm{m} 1$ & $\mathrm{~m} 2$ & $\mathrm{~m} 3$ & $\mathrm{~m} 4$ & $\mathrm{~m} 5$ \\
\hline K1 (MPa) & 27.1675 & 32.84006 & 35.049762 & 35.8090186 & 59.8890943 \\
\hline $\mathrm{K} 2(\mathrm{MPa})$ & 964.286 & 586.9565 & 790.2439 & 3056.60377 & 733.031674 \\
\hline$\eta(\mathrm{MPa} \cdot \mathrm{s})$ & $5.4 E+07$ & 67363268 & 86390175 & 351439009 & 63883287.3 \\
\hline
\end{tabular}

When $t \longrightarrow \infty, \varepsilon_{\infty}=\sigma_{0} / K_{1}+\sigma_{0} / K_{2}, \quad \varepsilon_{0}$ is obtained, where $\varepsilon_{\infty}$ denotes that finally the creep tends to be stable and is the sum of instantaneous deformation of two Hooke bodies.

During the creep test, the $\varepsilon$ - $t$ relation of fragmentized coals with different grain sizes is shown in Figure 6.

The analysis of the $\varepsilon$ - $t$ relation during the creep test of fragmentized coals and the creep model parameters of fragmentized coals with different grain sizes are shown in Table 3.

4.2. Reasonability Analysis of the Creep Constitutive Model of Fragmentized Coals. The constitutive equation of fragmentized coals with different grain sizes can be obtained by the Kelvin-Voigt model and based on the material creeping 
TABLE 4: Fitting parameter table of the first index decay of fragmentized coals with different grain sizes under the stress of $12 \mathrm{MPa}$.

\begin{tabular}{lccccc}
\hline Parameter grain size & $\mathrm{m} 1$ & $\mathrm{~m} 2$ & $\mathrm{~m} 3$ & $\mathrm{~m} 4$ & $\mathrm{~m} 5$ \\
\hline A & -0.0123 & -0.02073 & -0.01528 & -0.0397 & -0.01618 \\
B & 5.48016 & 10.01032 & 9.60959 & 10.76907 & 9.20648 \\
C & 0.45403 & 0.38592 & 0.35751 & 0.33906 & 0.21664 \\
\hline
\end{tabular}

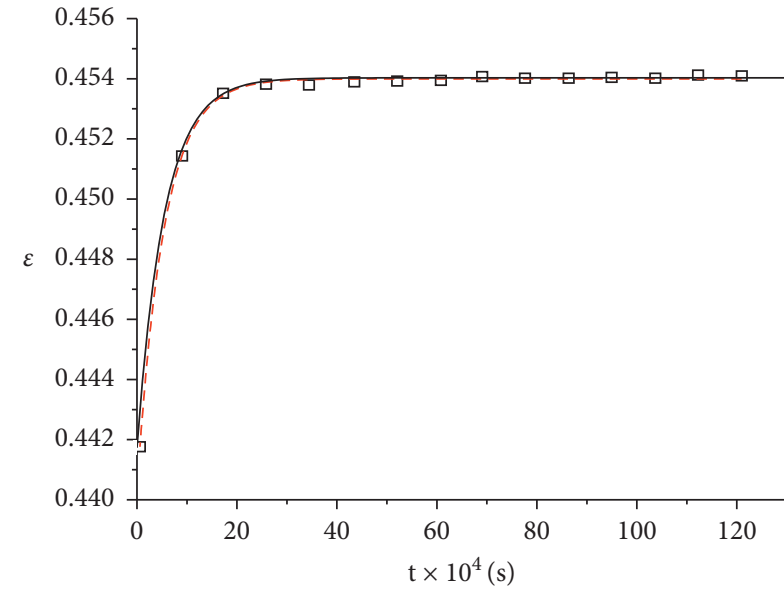

$\square \quad$ Real measuring points of creep test

- - Fitting curve of first index decay

- Creep curve of Kelvin-Voigt model

(a)

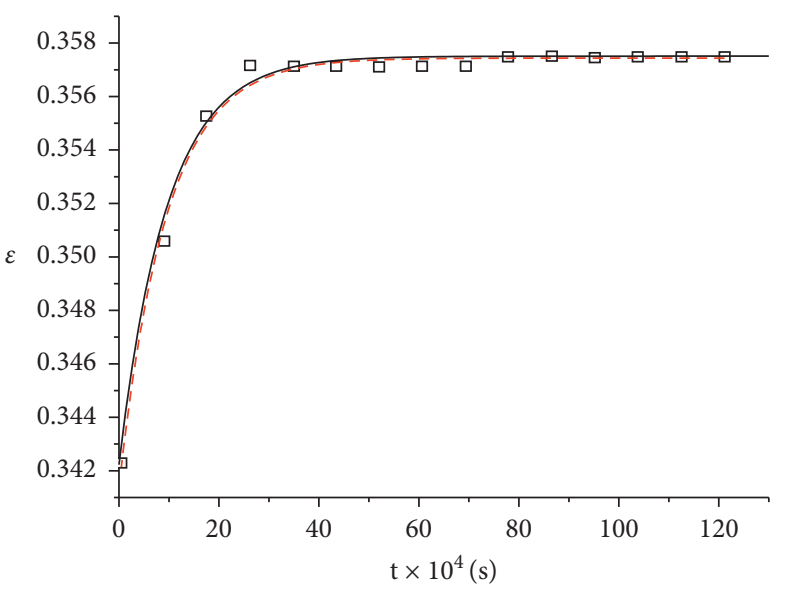

ㅁ Real measuring points of creep test

- - Fitting curve of first index decay

_ Creep curve of Kelvin-Voigt model

(c)

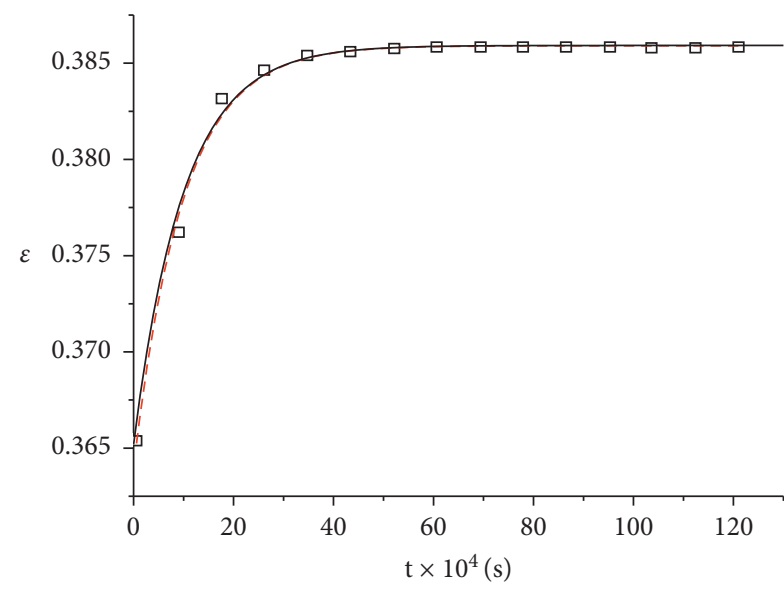

- Real measuring points of creep test

- - - Fitting curve of first index decay

_ Creep curve of Kelvin-Voigt model

(b)

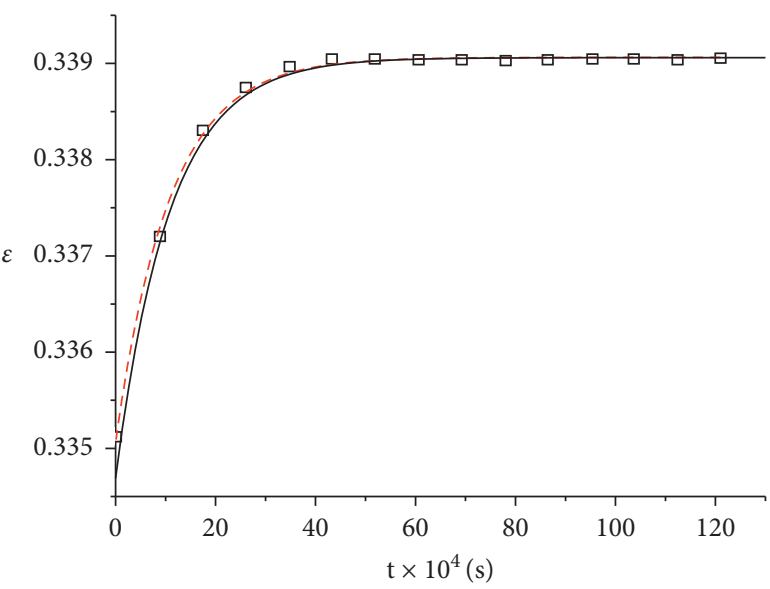

Real measuring points of creep test

- - Fitting curve of first index decay

_ Creep curve of Kelvin-Voigt model

(d)

FIgURE 7: Continued. 


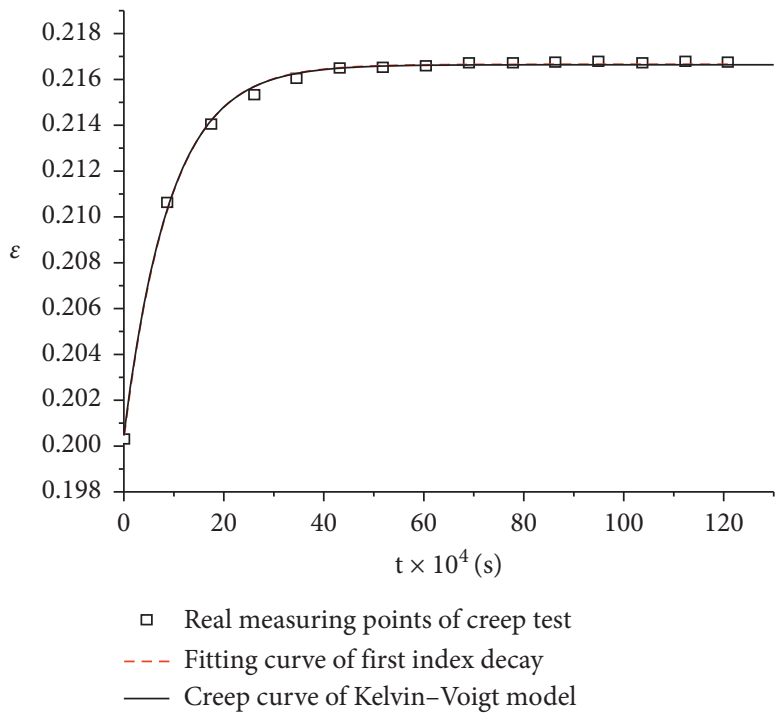

(e)

Figure 7: Kelvin-Voigt model and the fitting curve of first index decay of fragmentized coals with different grain sizes. (a) m1. (b) m2. (c) m3. (d) m4. (e) m5.

TABle 5: Comparison of correlation coefficients of the Kelvin-Voigt model and the fitting curve of first index decay of fragmentized coals with different grain sizes.

\begin{tabular}{lccccc}
\hline Grain size correlation coefficient & $\mathrm{m} 1$ & $\mathrm{~m} 2$ & $\mathrm{~m} 3$ & $\mathrm{~m} 4$ & $\mathrm{~m} 5$ \\
\hline Exponential decay curve fitting & 0.99937 & 0.99549 & 0.99439 & 0.99866 & 0.99835 \\
Kelvin-Voigt creep model & 0.99965 & 0.995799 & 0.9954751 & 0.9988852 & 0.9989241 \\
\hline
\end{tabular}

property of fragmentized coals. Hereby, the reasonability and precision of the Kelvin-Voigt model need to be further analyzed.

In the past research studies on the creeping property of fragmentized rocks, the exponential decay fitted equation is mostly adopted to describe the creep process of fragmentized rocks. According to the analysis of the $\varepsilon$ - $t$ relation of fragmentized coals with different grain sizes as shown in Figure 6, the fitted equation of the first index decay $\varepsilon$ - $t$ can be obtained:

$$
\varepsilon=A \cdot \exp \left(-\frac{t}{B}\right)+C
$$

Parameters of the fitted equation of the first index decay $\varepsilon$ - $t$ are shown in Table 4 .

Kelvin-Voigt model and the fitting curve of first index decay of fragmentized coals with different grain sizes are shown in Figure 7.

On the basis of creep test data of the saturated fragmentized coals and through the statistical correlation analysis and calculation according to the Kelvin-Voigt model and the fitting curve of the first index decay of fragmentized coals with different grain sizes, their correlation coefficients are shown in Table 5.
As shown in Table 5, according to the creep constitutive relation of fragmentized coals described by the creep model, the curve correlation coefficient at all grain sizes reaches above 0.995; at all grain sizes, the correlation coefficient between the Kelvin-Voigt creep model of fragmentized coals and test data is larger than the past fitting curve of the first index decay, indicating that compared with the fitting curve of the first index decay, the creep constitutive equation of fragmentized coals established by the Kelvin-Voigt model is more precise, and the latter is reasonable; in addition, the creep constitutive equation of fragmentized coals established by the Kelvin-Voigt model can explain the creep test process of saturated fragmentized coals from the perspective of the material property of fragmentized coals.

\section{Conclusions}

According to the analysis of the porosity variation laws of fragmentized coals with five grain sizes during creep process under two stresses and its creep constitutive relation, the following conclusions are drawn:

(1) Under two stresses, the porosity logarithm of fragmentized coals during creep shows a linear 
negative correlation with the time: $\ln (n-a)=-$ $\mathrm{ct}+\operatorname{lnb}$.

(2) The porosity decrease is evidently divided into three phases. Phase I: slippage, malposition, and deformation occur between particles of fragmentized coals, and porosity decreases quickly. Phase II: slippage and malposition seldom happen to be fragmentized coal particles, and the fragmentized coal particles form a self-supporting structure; as the stress continues to be loaded, under the squeezing impact, the porosity changes relatively quickly and tends to decrease. Phase III: due to the attrition crushing in Phase II, a larger gap exists between coal particles and is filled with fine coal particles after fragmentized. As the stress continues to be loaded, the gap between particles becomes smaller and is difficult to be filled; thus, the entire state of coal particles tends to be a critical stable skeleton structure, and deformation tends to be stable.

(3) When the stress level is lower, the porosity decreases slowly; when the stress level rises up, the porosity decreases quickly; finally when the stress level remains stable, the porosity is smaller.

(4) Under the equal stress, as the grain size of fragmentized coal decreases, the porosity tends to decrease, and as the grain size of fragmentized coal tends to stabilize, the porosity tends to increase.

(5) The creep constitutive equation of fragmentized rocks with different grain sizes was established by using the Kelvin-Voigt model; the correlation analysis shows that the curve correlation coefficient reaches above 0.995 , and at various grain sizes, the Kelvin-Voigt creep model and test data correlation coefficient are larger than the past fitting curve of the first index decay, indicating the Kelvin-Voigt creep model of fragmentized coals is reasonable.

\section{Data Availability}

The data used to support the findings of this study are included within the article.

\section{Conflicts of Interest}

The authors declare that there are no conflicts of interest regarding the publication of this paper.

\section{Acknowledgments}

This work was supported by the Fundamental Research Funds for the Central Universities (Grant no. 2019QNA19).

\section{References}

[1] Xu Jiang, LuQi, X. Wu, and D. Liu, “The fractal characteristics of the pore and development of briquettes with different coal particle sizes," Journal of Chongqing, vol. 34, pp. 81-89, 2011, in Chinese.
[2] J. E. Turney, Subsidence above Inactive Coal Mines: Information for the Homeowner, Colorado Geological Survey, Colorado Mined Land Reclamation, Division Inactive Mine Reclamation Program, and Department of Natural Resources, Golden, CO, USA, 1985.

[3] C. Zhang, J. Liu, Y. Zhao, P. Han, and L. Zhang, "Numerical simulation of broken coal strength influence on compaction characteristics in goaf," Natural Resources Research, vol. 29, no. 4, pp. 2495-2511, 2020.

[4] S. Li, "Stability study of fluid-solid coupled dynamic system of seepage in accumulative broken rock," Arabian Journal of Geosciences, vol. 13, no. 14, 2020.

[5] C. Zhang and L. Zhang, "Permeability characteristics of broken coal and rock under cyclic loading and unloading," Natural Resources Research, vol. 28, no. 3, pp. 1055-1069, 2019.

[6] M. Zhan-guo, G.-L. Guo, R.-H. Chen, and M. Xian-biao, “An experimental study on the compaction of water-saturated over-broken rock," Chinese Journal of Rock Mechanics and Engineering, vol. 24, no. 7, pp. 1139-1144, 2005, in Chinese.

[7] C. H. Scholz, "Mechanism of creep in brittle rock," Journal of Geophysical Research, vol. 73, no. 10, pp. 3295-3302, 1968.

[8] H. W. Zhou, L. Zhang, X. Y. Wang, T. L. Rong, and L. J. Wang, "Effects of matrix-fracture interaction and creep deformation on permeability evolution of deep coal," International Journal of Rock Mechanics and Mining Sciences, vol. 127, p. 104236, 2020.

[9] M. Li, J. Zhang, G. Meng, Y. Gao, and A. Li, "Testing and modelling creep compression of waste rocks for backfill with different lithologies," International Journal of Rock Mechanics and Mining Sciences, vol. 125, p. 104170, 2020.

[10] P. N. Hagin and M. D. Zoback, "Viscous deformation of unconsolidated sands-part 1: time-dependent, frequency dispersion, and attenuation," Geophysics, vol. 69, no. 3, pp. $653-861,2004$.

[11] W. He, A. Hajash, and D. Sparks, "Creep compaction of quartz aggregates: effects of pore-fluid flow--A combined experimental and theoretical study," American Journal of Science, vol. 303, no. 2, pp. 73-93, 2003.

[12] F. M. Chester, J. S. Chester, A. K. Kronenberg, and A. Hajash, "Subcritical creep compaction of quartz sand at diagenetic conditions: effects of water and grain size," Journal of Geophysical Research, vol. 112, no. B6, p. B06203, 2007.

[13] A. K. Parkin, "Creep of rockfill," in Advances in Rockfill Structure, pp. 221-239, Kluwer Academic Publishers, London, UK, 1992.

[14] Z. Ma, P. Wang, Z. Guo-zhen, and K. Sun, "Test study on the changing of the porosity for water-saturated granular shale during its creep," in Proceedings of the 44th US Rock Mechanics Symposium and the 5th US/Canada Rock Mechanics Symposium, Salt Lake City, UT, USA, June 2010. 